\title{
Melanoma Detection From Lesion Images Using Optimized Features Selected by Metaheuristic Algorithms
}

\author{
Soumen Mukherjee, RCC Institute of Information Technology, India \\ (iD) https://orcid.org/0000-0002-8211-0507 \\ Arunabha Adhikari, West Bengal State University, India \\ Madhusudan Roy, Saha Institute of Nuclear Physics, India
}

\begin{abstract}
This paper deals with a simple but efficient method for detection of deadly malignant melanoma with optimized handcrafted feature sets selected by three alternative metaheuristic algorithms, namely particle swarm optimization (PSO), ant colony optimization (ACO), and simulated annealing (SA). A total of 1,898 features relating to lesion shapes, colors, and textures are extracted from each of the 170 non-dermoscopy camera images of the popular MED-NODE dataset. This large feature set is then optimized, and the number of features is reduced up to the range of single digit using metaheuristic algorithms as feature selector. Two well-known supervised classifiers (i.e., support vector machine $[\mathrm{SVM}]$ and artificial neural network [ANN]) are used to classify malignant and benign lesions. The best classification accuracy result found by this method is $87.69 \%$ with only seven features selected by PSO using ANN classifier which is far better than the results found in the literature so far.
\end{abstract}

\section{KEYWORDS}

Ant Colony Optimization, Feature Selection, Med-Node, Melanoma, Metaheuristic, Multilayer Perceptron, Particle Swarm Optimization, Simulated Annealing

\section{INTRODUCTION}

Skin cancer is a common and lethal type of cancer and in recent years has become prevalent mainly in western countries of the world (Leiter, Eigentler, \& Garbe, 2014). The most common causes of skin cancer are exposure of ultraviolet rays, urban lifestyle, clothing pattern, thin ozone layer, genetic predisposition etc. (Leiter, Eigentler, \& Garbe, 2014). There are two major categories of skin cancer i.e., melanoma and non-melanoma. Skin cancers are found at a higher occurrence rate in white skinned individuals than the darker skinned persons. The skin biopsy is the best way to confirm skin malignancy and it is therefore taken as the gold standard. However, it costs around $\$ 100$ to $\$ 300$. In the category of non-melanoma type skin cancer, the occurrence rates of Basal and Squamous cell

\section{DOI: 10.4018/IJHISI.288542}

This article published as an Open Access article distributed under the terms of the Creative Commons Attribution License (http://creativecommons.org/licenses/by/4.0/) which permits unrestricted use, distribution, and production in any medium, provided the author of the original work and original publication source are properly credited. 
carcinoma are the highest, found in more than $80 \%$ of the malignant skin cancer cases (Apalla, Lallas, Sotiriou, Lazaridou, \& Ioannides, 2017). Recently, malignant melanoma is becoming an epidemic mainly in countries like Australia and New Zealand. It is the fastest growing skin cancer (4\% to 7\% per year) in Europe from the mid $20^{\text {th }}$ century. The cost of treatment of melanoma is very high with the United States alone recording a 288\% increase in the last 10-15 years (Apalla, Lallas, Sotiriou, Lazaridou, \& Ioannides, 2017). The total direct and indirect cost involved in prevention and cure of melanoma disease has increased up to 7 billion dollars worldwide, in the recent years (Matthews, et al., 2017). Dermatologist traditionally use several lesion scoring techniques like ABCD rule, 7-point checklist etc. for primary indication of the possibility of malignancy and to identify malignant melanoma from other skin lesions (Aurora, Begoña, \& Carmen, 2014). These scoring methods are widely used by medical experts before referring to skin biopsy. The accuracy levels of detection for malignant melanoma by the above mentioned semi-quantitative methods are limited. Though expert dermatologists can detect melanoma or non-melanoma type of skin cancer in early stages, the detection accuracy is about $80 \%$ (Morton, \& Mackie, 1998). In a benchmark skin cancer research work of melanoma classification, using only the domain knowledge of dermatologists, Brinker et al. (Brinker, Hekler, et al., 2019) in the year 2019 have shown that sensitivity and specificity can reach up to of $74.1 \%$ and $60.0 \%$ respectively, when the diagnosis is from inspecting dermoscopy images by the naked eye. Sensitivity and specificity were shown to reach even up to of $89.4 \%$ and $64.4 \%$ when non-dermoscopy images were used. In their work, Brinker et al. have done an overall survey in 12 German university hospitals with 157 dermatologists. The dermatologists have varied level of practical experience, position, sex and age. In the survey each dermatologist had images as input and they had to label the lesion in the image as malignant or benign. They used two well known datasets, (i) ISBI 2016 challenge dermoscopy dataset and (ii) MED-NODE non-dermoscopy dataset. In recent times for the advancement of image acquisition process dermoscopy is expected to enhance malignancy detection chances but it is found from their experiment that non-dermoscopy datasets gives significantly better sensitivity and specificity than the dermoscopy datasets. On the other side in the last two decades there is a phenomenal growth in computer aided skin cancer detection research. Using computer vision and pattern recognition system with the help of machine learning and recent deep learning technologies it is much easier and less costly to categorize the skin lesion as malignant or benign.

In the present work aiming identification of malignant melanoma skin cancers non-dermoscopy camera images are collected from a standard secondary data source (MED-NODE) and with the help of image processing, soft computing and machine learning the images are analyzed and classified. At first a considerable number of hand-crafted features are extracted from images of the dataset and then these features are used to classify the malignant and benign lesions using two popular supervised algorithms Support Vector Machine (SVM) and Artificial Neural Network (ANN). In this work, from the MED-NODE skin cancer dataset total 1898 features are extracted from the segmented (using Otsu algorithm) skin lesion area. As a large number of features may contain noise, a smaller but effective (which results in higher classification accuracy) features set is sought after. Selection of the best set of features from a large set by exhaustive search requires exponential time and therefore is not possible most of the time. To solve this problem metaheuristic algorithms are used (Yeh \& Chan, 2017). In the present work three metaheuristic algorithms namely PSO, ACO and SA are used to select an optimized set of features from a big set of hand crafted features. PSO feature selection algorithm selects optimized feature sets (only 7 out of 1898 features) to provide best classification accuracy of $87.69 \%$. It is also found that texture features are best for skin lesion classification. In the present work two classification algorithms are used i.e. SVM and ANN. The classification results found from the ANN classifier are better than SVM classifiers with mostly all sets of features. The result of the present paper shows that higher accuracy is achieved with less than $0.4 \%$ of all the features selected by the above mentioned optimizations. PSO and ACO are simple, fast and global search approaches whereas SA has a good convergent property and is relatively free from local optima. It is observed 


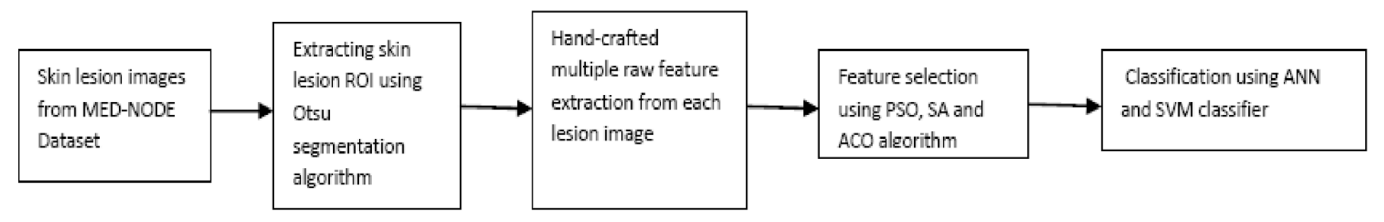

in the proposed work that the PSO feature selection algorithm is much better than the ACO and SA feature selection algorithms. Figure 1 shows the signal processing flow diagram of the whole work.

The paper is organized in the following sequence. In the next section relevant literature is surveyed with a additional subsection on the use of metaheuristic algorithms for feature selection. In the subsequent sections the methodology, MED-NODE dataset and the extracted features adopted are described following a short description of the specific metaheuristic used in this article. Result analysis and result comparison with related work follows in a separate section. Finally the conclusion section is given with the future direction of the research.

\section{LITERATURE SURVEY}

Computer aided skin cancer detection is a new area of research. This section is further divided into three sub-sections. In the first subsection skin cancer classification including using standard dermoscopy and non-dermoscopy datasets other than MED-NODE, are discussed. A separate section is written for the works on melanoma classification using only MED-NODE datasets; finally related works on metaheuristic feature selection are discussed.

\section{Skin Cancer Classification Using Dataset Other Than MED-NODE}

In this section some related works using dataset other than MED-NODE are given. In this section at first research findings with hand-crafted features are discussed and then research work with Deep learning are given in ascending order of classification accuracies separately.

With traditional features, Laskaris (Laskaris, Ballerini, Fisher, Aldridge, \& Rees, 2010) and McDonagh (McDonagh, Fisher, \& Rees, 2008) using Dermofit dataset classified into the malignant and benign lesions and achieved classification accuracy of $80.64 \%$ and $83.7 \%$ using 31 and 234 images respectively. Mukherjee et al. (Mukherjee, Adhikari, \& Roy, 2018d) done a work with only top level 163 features, where all the 163 features have highest ranks in four types of different feature ranking algorithms and found a classification accuracy of $86.2 \%$ is found using Dermofit dataset. Tan et al. (Tan, Zhang \& Jiang, 2016) achieved 88\% classification accuracy with Dermofit dataset consisting of 1300 images with SVM classifier. Lesion image pre-processing like skin hair removal, contrast enhancement were done for every image. Total 1472 features from the extracted 3914 features were optimized using Genetic Algorithm (GA). Using best ranked 50 features of University of Waterloo skin cancer dataset (206 images) classification accuracy of $87.1 \%$ by 1 hidden-layer Artificial Neural Network (ANN) and an accuracy of $88.38 \%$ by 2 hidden-layer ANN (neuron number optimized by Particle Swarm Optimization) is found by Mukherjee et al. (Mukherjee, Adhikari, \& Roy, In Press). Thanh-Toan et al. (Thanh-Toan, et al., 2018) used the Singapore national skin centre database with 184 lesion images including 67 images of malignant melanoma. The acquired images had different resolution, focusing, size, extra body parts. So, the images required good segmentation before classification. A separate segmentation algorithm was proposed by them with $80.09 \%$ accuracy, which gave better accuracy than the Otsu and MST segmentation algorithm. For classification of malignant and benign lesions 54 color features, 16 border features and 9 texture features were separately used 
and $92.09 \%, 77.64 \%$ and $80.61 \%$ accuracy were found with color, border and texture respectively for the above mentioned datasets. Gorai et al. (Gorai \& Chatterjee, 2019) have done classification work of a total 7201 dermoscopic images including 2879 melanoma images taken mainly from Dermoscopic Atlas, ISIC and PH2 dataset. They have generated a set of uniform size kernels from sample images by manual extraction of specific regions of the lesion. These kernels have color and morphological feature descriptors. These featured descriptors are used to get the correlated feature regions of the input images. Then cross-correlation of images with the selected kernels has been identified. An ensemble multiclass classification procedure binary SVM classifiers with RBF kernel is used in the work with 70:30, training: testing ratio. They have achieved $98.76 \%$ sensitivity.

With the advent of Deep learning recently more and more researchers are shifting their interest in this domain. Esteva et al. (Esteva, et al., 2017) used Deep Convolution Neural Network (CNN) network for malignancy and benign classification. A huge number of 129450 images were used in their publication in Nature. They designed classification models belonging to three classes namely malignant, benign and non-neoplastic. The classification accuracy achieved by them is $72.1 \%$, and with the same dataset the dermatologists with their clinical judgment achieved around $65 \%$ accuracy. Codella et al. (Codella, et al., 2017) achieved a classification accuracy of $76 \%$ using Deep learning architecture using skin cancer dataset. With Dermofit image dataset of University of Edinburgh, consisting of 1300 images an accuracy of $84 \%$ for malignancy detection was achieved by Li et al. (Li, et al., 2016). In their work they created a system where at first the system detects malignant lesions and then tracks the lesion within the complete human body image. They also used two nonexpert humans with training on the same dataset and estimated the accuracy of $82 \%$ and $85 \%$. Valle et al. (Valle, Fornaciali, Menegola \& Tavares, 2017) performed an extensive analysis of melanoma screening. They collectively used different skin cancer datasets like ISIC Archive, EDRA Dermoscopy Atlas, Dermofit and PH2. They used two pre-trained Deep learning networks ResNet, and Inception. They achieved 87.4\% classification accuracy. Mukherjee et al. (Mukherjee, Adhikari, \& Roy, 2018c) did a work with deep CNN with 10 fold data augmentation using Dermofit image datasets, which yields an accuracy of $90.58 \%$. Xiao et al. (Xiao \& Wu, 2020) have done classification work with total 2000, 150 and 600 training, validation and test dermoscopic images respectively taken from ISIC dataset. They have segmented each image to find the salient region and then identified local blocks from the segmented lesion. Local Binary Pattern and Deep Convolution features are extracted from both segmented and local blocks. They have used two popular deep neural network models i.e. DenseNet-121 and ResNet-50 in their work. With ResNet-50 they have achieved 84.8\% accuracy.

\section{Melanoma Classification Using MED-NODE Dataset}

In this section the works related to MED-NODE dataset is presented in ascending order of classification accuracies found. Spiros et al. (Spiros, et al., 2017) worked out two classifications of benign mole and malignant melanoma from a camera image database collected from multiple sources with 14 numbers number of texture, shape and color features. They achieved accuracy of $76.20 \%$, sensitivity of $73.90 \%$ and specificity of $77.80 \%$. Mahecha et al. (Mahecha, Parra \& Velandia, 2018) found an accuracy level of $76.67 \%$ with 730 images collected from both MED-NODE and ISIC projects. In their work they have done several pre-processing of input lesion images like illumination adjustment, Gaussian filtering, and conversion to gray-scale before using lesion segmentation. Finally they have used six layers of convolutional neural network (CNN) for classification work. Sultana et al. (Nazneen, Mandal, \& Puhan, 2018) in their work used three dataset ISBI 2016, MED-NODE and PH2 where they have used a deep architecture consisting of 50 layers ResNet pre-trained on ImageNet, and for the purpose of tuning some of the final fully connected layers are retrained again. Each image in the input layer is resized into a $224 \times 224$ pixel matrix. The dataset is augmented by rotation, translation and flipping of the image. The system achieves a maximum accuracy of $77.1 \%$ with MED-NODE dataset. Jafari et al. (Jafari, Samavi, Karimi, Soroushmehr, Ward, \& Najarian, 2016) worked on 170 MED-NODE image dataset. They have pre-processed each image by noise removal and illumination correction 
before border detection and segmentation. After segmentation they have extracted 10 features from each image. They have used SVM classifiers for the two class classification problem with $75 \%$ data for training set and rest for testing set. Jafari et al. have achieved 90\%, 72\% and 79\% sensitivity, specificity and accuracy respectively. Giotis et al. (Giotis, Molders, Land, Biehl, Jonkman, \& Petkov, 2015) extracted 675 features each from 170 MED-NODE dataset images to achieve $81 \%$ of classification accuracy. Each image is pre-processed to eliminate uneven lighting and focusing problems of the camera. In their work CLAM classifier is used. Present authors in a previous communication extracted 1865 features from MED-NODE and with top 10 features ranked by ReliefF ranking algorithm and with top 25 principal component features a classification accuracy of $83.33 \%$ and $87.18 \%$ is found respectively using Multilayer Perceptron (MLP) with a single hidden layer (Mukherjee, Adhikari, \& Roy, 2020). In a subsequent work, the same group of authors (Mukherjee, Adhikari, \& Roy, 2018a) has achieved a higher accuracy of $85.9 \%$ when instead of feature selection the entire set of 1875 features was used in a two hidden layers MLP where they optimized number of neurons in the hidden layers by a metaheuristic called PSO. In another work Mukherjee et al. (Mukherjee, Adhikari, \& Roy, 2018b) achieved $91.02 \%$ classification accuracy with 1886 number of features where neuron number in the two hidden layers are optimized by PSO and SA. The main differences between the work of Mukherjee et al. (Mukherjee, Adhikari, \& Roy, 2018a) and Mukherjee et al. (Mukherjee, Adhikari, \& Roy, 2018b) is that in the former work for texture feature extraction 4 and 8 levels of quantization are used and the personal learning rate of PSO algorithm is same with social learning rate. In the later work (Mukherjee, Adhikari, \& Roy, 2018b) for texture feature extraction 64 and 128 quantization levels were used and personal learning rate was made multiple of 2000 than social learning rate for PSO algorithm. In table 5 the comparison of the related work result is given with corresponding classification accuracies.

\section{Metaheuristic Feature Selection}

Some of the metaheuristic algorithms are inspired from nature and are widely used in machine learning for feature selection or optimization. Quite a few metaheuristic algorithms are non-bio inspired also. Some of the research works are discussed in this section. Fong et al. (Fong, Wong, \& Vasilakos, 2016) used Correlation based Feature Selection, PSO and Accelerated PSO algorithms for feature selection with 5 datasets of different domains collected from UCI archive. It is observed that with Naïve-Bayes, Decision Tree, SVM and ANN classifier a 12.76\%, 1.93\%, $2.11 \%$ and $0.3 \%$ increase in classification accuracy is found with only $64 \%, 26 \%, 26 \%$ and $64 \%$ of the total features in comparison with all features. Seijas et al. (Seijas, et al., 2015) used three metaheuristic algorithms Binary Fish School Search (BFSS), Advanced Binary ACO and Binary PSO to classify MNIST handwritten dataset with SVM Classifier. With BFSS, ABACO and BPSO 93.36\%, 90.15\% and $92.08 \%$ classification accuracy is achieved with only $41 \%, 22.4 \%$ and $27 \%$ of the total features. Yeh and Chan (Yeh, Chan, 2017) used 69 benign, 54 malignant and 68 normal mammograms from the MIAS database and extracted a total of 277 features. They have used Genetic Algorithm (GA), ACO, SA and PSO for feature selection. With only 189 numbers of features found by PSO a classification accuracy of $96.9 \%$ is found. Naeini et al. (Naeini, Babadi, Mirzadeh, \& Amini, 2018) have worked on 2 datasets of high resolution satellite image collected from WorldView-2 sensor. Total 173 features are mined from satellite images. The Object Based Feature (OBF) selection is done by PSO, GA, Artificial-Bee-Colony, and Honey-Bee-Mating algorithms. It is found from their work that PSO is a low cost method for identifying different objects like trees buildings, roads etc. Ighazran et al. (Ighazran, Alaoui, \& Boujiha, 2018) have done a review on metaheuristic feature selection in sentiment analysis. In some research work more than one metaheuristic algorithm is merged together to create a new hybrid algorithm, which may perform better in feature selection. Arora et al. (Arora, Singh, Sharma, Sharma, \& Anand, 2019) designed an algorithm by hybridization of Grey-Wolf and Crow search algorithm. They have used a total of 23 standard benchmark uni-modal, multi-modal and 
fixed-dimensional multi-modal mathematical functions and 21 data sets and compared the result of the new hybrid algorithm with 10 popular metaheuristic algorithms as feature selector.

\section{RESEARCH CONTRIBUTION OF THE PAPER}

In this work three metaheuristic algorithms i.e. PSO, ACO and SA are used to select an optimized set of features. Out of these algorithms PSO selects optimized feature set to provide best classification accuracy of $87.69 \%$. It is also found that texture features are best for skin lesion classification. The classification results found from the ANN classifier are better than SVM classifier with mostly all sets of features. In this work it is found that higher accuracy is achieved with less than $0.4 \%$ of all the features selected by the above mentioned optimizations.

\section{METHODOLOGY}

The MED-NODE dataset consists of 70 malignant and 100 benign images. Lesions are segmented using masks generated by Otsu algorithm (Otsu, 1979). For the masks MED-NODE images are converted to gray-scale images which are typically bimodal in nature and Otsu algorithms then decide the necessary threshold (Mukherjee, Adhikari, \& Roy, 2020). With the help of the mask the minimal box bounding the lesion is cropped from the image. This ensures maximal background removal. The remaining background is set to zero pixels. Total 1898 skin lesion feature values relating to shape, lesion color (Pereira, Marco, Frade, Rangaraj, \& Azevedo-Marques, 2013) and texture are calculated from each segmented lesion image. A total of 10 shape features, 36 color features and 1852 texture feature values are extracted from each segmented lesion. The texture features include 88 number of Gray-Level- Run-Length-Matrix (GLRLM) (Tang, 1998) and 1764 number of GrayLevel-Co-Occurrence-Matrix (GLCM) (Haralick, Shanmugam, \& Dinstein, 1973; Soh, \& Tsatsoulis, 1999; Clausi, 2002). The types, categories and names of all the features used in the work are given in the next section. Among the 1898 features which are directly extracted from individual images of MED-NODE dataset, 12 features are disqualified and rejected as they show little or no variation throughout the feature set. Finally, all the experimentation is done on the remaining 1886 features. Three metaheuristic algorithms i.e. PSO, ACO and SA algorithms are used to select optimized feature sets. Using these optimized feature sets classification is done using SVM and ANN classifiers. In figure 1 and figure 3 flow diagram of the proposed work is shown which describes the whole work.

\section{MED-NODE DATASET}

There are several secondary standard image dataset of dermoscopy and non-dermoscopy available online for melanoma and non-melanoma skin cancer. Some of them are Dermofit, PH2, MED-NODE, Atlas of Dermoscopy, DermNet and ISIC. Among them MED-NODE (Giotis, Molders, Land, Biehl, Jonkman, \& Petkov, 2015) is a publicly available standard melanoma dataset consisting of a total 170 malignant melanoma and benign nevi lesion images. These are non-dermoscopy camera images (.jpeg format with different pixel dimensions) archived in Dermatology department, Groningen University. Each image in the MED-NODE dataset (Giotis, Molders, Land, Biehl, Jonkman, \& Petkov, 2015) is taken by Nikon camera with 2.8/105 mm lens. An average $330 \mathrm{~mm}$ distance within the camera and the lesion is maintained throughout the dataset. The ground truth for malignant melanoma images of the MED-NODE database is verified by biopsy and benign nevi are confirmed by the expert dermato-pathologist and indexed as "gold-standard". MED-NODE lesion images are categorized into 100 malignant melanoma and 70 benign nevi by Groningen University. In the proposed work a two-class binary (malignant and benign) classification is performed by the standard and considerably balanced MED-NODE database. 
Figure 2. (a) and (b) Malignant melanoma images from MED-NODE
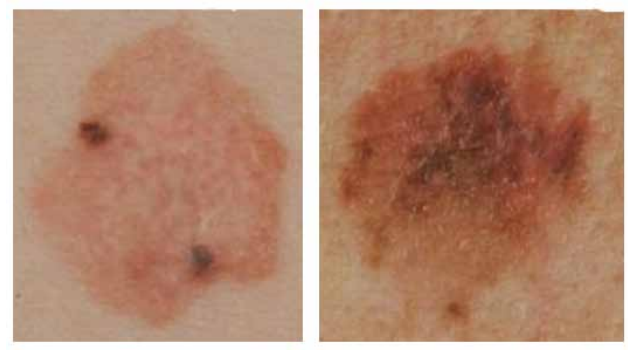

Figure 3. (a) and (b) Benign images from MED-NODE
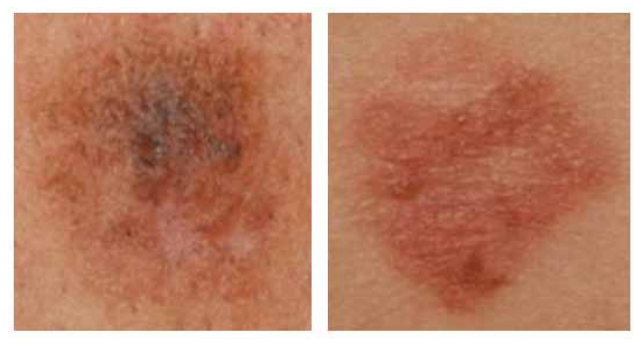

Sample malignant melanoma and benign images are given in figure 2 and figure 3. West Bengal State University's (West Bengal, India) Institutional Ethics Committee (IEC) has given ethical approval for using the dataset in this work.

\section{EXTRACTED FEATURE SET DETAILS}

From the total extracted 1898 features, the number of color features are 36 (Pereira, Marco, Frade, Rangaraj, \& Azevedo-Marques, 2013), structural shape features are 10 and there are 88 and 1764 number of GLRLM and GLCM features respectively. "RGB", "HSV", "YCbCr",'Lab",'YIQ" and "XYZ" color models are used in 3 of their individual color channels for mean and standard deviation features. The features extracted from the skin lesion image are so chosen to comply with the skin lesion scoring techniques (mentioned in the Introduction section) like ABCD, 3-point checklist, 7-point check list etc, Lesion shape features are important for representing the Asymmetry (A), Border (B) and architecture property of a lesion. Lesion color features are important for representing the Color (C) property of a lesion. Lesion texture features are important for representing the Differential (D) structure, pigment network, dots and streak property of a lesion. For calculating several second order GLCM \& GLRLM feature values, various statistical two pixel joint probability distributions are taken into account. Using GLRLM, distribution of the number of consecutive same gray-level pixels with " $i$ " intensity in angle $\theta^{\circ}$ in the image can be found. From GLRLM, 11 second order statistical features are extracted for two quantization levels with four angles. Using GLCM, number of "i" intensity gray-level pixels with a distance $d$ and angle $\theta$ with respect to another pixel of intensity " $\mathrm{j}$ " in the image can be found. From the GLCM matrix 21 second order statistical features are extracted with six inter-pixel distances and two quantization levels for $0^{\circ}$ angle. Color-GLCM features for 3 channels of RGB and HSV model with 2 quantization levels and 6 inter-pixel distances with an angle of $0^{\circ}$ are also calculated. Information of all features are given in table 1 . 
Table 1. Details of the 1898 features extracted from MED-NODE images (Mukherjee, Adhikari, \& Roy, 2020)

\begin{tabular}{|c|c|}
\hline $\begin{array}{l}\text { Feature Category \& Count } \\
\quad \text { (total } 1898 \text { features) }\end{array}$ & Feature Particulars \\
\hline $\begin{array}{l}\text { Structural Shape of the Lesion } \\
\text { (Feature No. 1-10) }\end{array}$ & $\begin{array}{l}\text { (F1)Area, (F2)Solidity, (F3)Perimeter, (F4)Circularity, (F5)Max Diameter, (F6)Min } \\
\text { Diameter, (F7,F8,F9,F10) Irregularity (of } 4 \text { types I,II,III,IV) }\end{array}$ \\
\hline $\begin{array}{l}\text { Skin Color of the Lesion } \\
(3 X 6 X 2 \text { i.e. } 36, \text { Feature No. } \\
11-46)\end{array}$ & $\begin{array}{l}\text { Three color channels with six color models ((I)RGB, (II)Lab, (III)XYZ, (IV)YCbCr, } \\
\text { (V)YIQ, (VI)HSV) and Two(2) features ((F1)Mean \& (F2)Standard Deviation) for } \\
\text { each }\end{array}$ \\
\hline $\begin{array}{l}\text { Texture GLRLM ( } 2 \text { X } 4 \text { X11 i.e. } \\
88, \text { Feature No. } 47-134)\end{array}$ & $\begin{array}{l}\text { Eleven features ((F1)Long-Run-Emphasis, (F2)Short-Run-Emphasis, (F3)Gray- } \\
\text { Level-Non-uniformity, (F4)Run-Percentage, (F5)Run-Length-Non-uniformity, (F6) } \\
\text { Low-Gray-Level-Run-Emphasis, (F7 \& F8)Short-Run-Low/High-Gray-Level- } \\
\text { Emphasis, (F9)High-Gray-Level-Run-Emphasis, (F10 \& F11)Long-Run-High/Low- } \\
\text { Gray-Level-Emphasis, for four angles }\left(135^{\circ}, 90^{\circ}, 45^{\circ} \& 0^{\circ}\right) \text { with two quantization } \\
(64 \& 128)\end{array}$ \\
\hline $\begin{array}{l}\text { Texture GLCM ( } 21 X 6 X 2 X 7 \text { i.e. } \\
\text { 1764, Feature No. } 135-1898)\end{array}$ & $\begin{array}{l}\text { Twenty one features ((F1)Auto-correlation, (F2)Contrast, (F3)Correlation, (F4)Sum- } \\
\text { of-Square, (F5)Sum-Variance, (F6)Sum-Average, (F7)Sum-Entropy, (F8)Cluster- } \\
\text { Prominence, (F9)Cluster-Shade, (F10)Dissimilarity, (F11)Energy, (F12)Entropy, } \\
\text { (F13)Max-Probability, (F14)Homogeneity, (F15)Difference-Variance, (F16) } \\
\text { Difference-Entropy, (F17 \& F18)Information-Measure-of-Correlation 1\&2, (F19) } \\
\text { Inverse-Difference-Normalized, (F20)Inverse-Difference, (F21)Inverse-Difference- } \\
\text { Moment-Normalized) for six inter-pixel distance (1,2,3,4,5,6) with two quantization } \\
\text { (64 \& 128) with seven formats (Three channels of "RGB", Three channels of "HSV" } \\
\text { and pure gray scale) for one angle }\left(0^{\circ}\right)\end{array}$ \\
\hline
\end{tabular}

\section{METAHEURISTIC ALGORITHMS}

The problem of using a bigger set of features is known as the "curse of dimensionality" which may introduce noise and degrade performance of the system. Reasons for selection of smaller subset of features include faster training, model complexity reduction, and improvement in model accuracy etc. In the literature several feature selection methods can be found e.g. feature ranking, feature transformation, feature subset selection, etc. In this proposed work 3 metaheuristic algorithms are employed for feature optimization. These are SA (Rutenbar, 1989; Fleischer, 1995), PSO (Kennedy, \& Eberhart, 1995) and ACO (Dorigo, Maniezzo, \& Colorni, 1996; Aurora, Begoña, \& Carmen, 2014). These algorithms are taken from two different categories; SA uses exploration of the whole search space whereas PSO and ACO make concerted exploitation (Wang, Zhang, \& Ji, 2015) of the search space. PSO and ACO are nature inspired population based algorithms but SA is a memory less, trajectory based single solution search method inspired from Physics. The authors have used metaheuristic candidates of different categories of algorithms to find the suitable methodology. Brief discussions on these three algorithms are given in the next subsection along with the values of the parameter used (https://yarpiz.com/).

\section{Simulated Annealing (SA)}

SA, proposed by Nicholas Metropolis (Rutenbar, 1989; Fleischer, 1995) is a probabilistic optimization algorithm which is intended to find the global optimum of the cost function when several local optima are present in the search space. The term "Annealing" is taken from the thermodynamics of metallurgy, which represents a process of heating a metal and cooling it slowly to increase the crystal size and to reduce crystal defect. A high temperature allows transition to a higher free energy state. The idea is borrowed in optimization problems where cost function is the analogue of free energy and transition to an inferior solution is quantified through a temperature like parameter. Initial high "temperature" allows a vivid investigation of the search space. The SA algorithm is used extensively to resolve optimization problems in the field of engineering and science. SA can be used for feature selection. 
It starts with selection of first $n_{s}$ features after a random ranking of total $n_{T}$ features. The ranking sequence is changed by small perturbation like random swapping, pop and insertion or partial inversion. Again first $\mathrm{n}_{\mathrm{s}}$ features are selected. Each set of selected features is associated with a cost (classification error) and the one with the least cost within a given number of iterations is declared as the final set of selected features.

\section{Particle Swarm Optimization (PSO)}

PSO iteratively progresses towards a better solution by parallel searches in terms of a population of solution seekers called "particles" whose instantaneous position in the search space represents a candidate solution (Kennedy, \& Eberhart, 1995). PSO finds the best solution progressively with iterations by moving each particle in a direction which is a weighted sum of the vectors along their individual instantaneous velocity, location of personal best position and also the location of the global best position. Each particle has an individual best-position learning rate and also a social learning rate. Location of each particle is revised in each iteration starting from a random value. Every particle position is controlled by its own personal best position (PBes) (by fitness function) and best position of the entire particles (GBes) present in the population. Using these values, the position $(P O S)$ and velocity (VEL) of the particle is revised using equation (1) and (2):

$$
\operatorname{POS}(t+1)=\operatorname{POS}(t)+V E L(t+1)
$$

where:

$$
V E L(t+1)=V E L(t)+a_{1} \cdot r a_{1} \cdot(\operatorname{PBes}(t)-\operatorname{POS}(t))+a_{2} \cdot r a_{2} \cdot(G B e s(t)-P O S(t))
$$

The values of the random number $r a_{1}$ and $r a_{2}$ varies between 0 and 1 and $a_{1}, a_{2}$ are the learning rates given in table 2 . The particle can have an inertia value which decides the contribution of the particle's previous velocity to its new velocity. The starting inertia is the inertia of the particle at the beginning. The inertia damping rate provides the change of inertia. The values used in the experiment are noted table 2 .

PSO can be used for selecting $n_{s}$ number of features from a total $n_{T}$ features. Particles are allowed to move in $n_{T}$ dimensional space and the features corresponding to the $n_{s}$ smallest components of the instantaneous position can be identified as selected features. It is to be noted that the axes of the search space represent the features and the values of the position vector along a particular axis is a kind of weight which determines its selection. Similar to SA the set of features with minimum cost is reported.

\section{Ant Colony Optimization (ACO)}

ACO is a metaheuristic algorithm which mimics the behavior of ants for their search of the optimum pathway from their home to the food supply and vice-versa (Dorigo, Maniezzo, \& Colorni, 1996; Aurora, Begoña, \& Carmen, 2014). When an ant leaves its nest for the search of food it leaves a trail of special chemical pheromone along the path it travels. An ant can also detect the intensity of the pheromone trail left by its forerunner. This indirect communication strategy is called "stigmergy" and this selects the shortest path from the nest to food. Each path has an initial level of deposited pheromone and rate of pheromone evaporation. ACO is useful to select a sequence of nodes from a graph where the edges are dynamically weighted by a factor called pheromone intensity which ensures that a frequently traversed edge is given more weightage. Movement of the ant generates a 
sequence. An ant at a node I will select a node $\mathrm{j}$ with a probability proportional to the pheromone intensity of the path $\mathrm{i}-\mathrm{j}$, i.e:

$$
p_{i j}=\frac{\tau_{i j}^{\alpha}}{\sum_{j} \tau_{i j}^{\alpha}}
$$

where, $\tau_{i j}=$ pheromone-intensity of edge $\mathrm{i}-\mathrm{j}$ and $\alpha$ is a constant known as ant pheromone-exponentialweight. The pheromone updating equation is:

$$
\tau_{i j}=(1-\rho) \tau_{i j}+\Delta \tau_{i j}
$$

where, $\rho=$ pheromone-evaporation-rate and $\Delta \tau_{i j}=$ pheromone-deposition in the path defined as $\Delta \tau_{i j}=\sum_{k} \frac{1}{L_{k}}$. Here $L_{k}$ is the cost of the $\mathrm{k}^{\text {th }}$ path which passes through the edge $\mathrm{i}-\mathrm{j}$. ACO can be used for selecting $n_{s}$ number of features from a total $n_{T}$ features. Each feature can be considered as a node of a graph and the first $n_{s}$ nodes of the ACO sequence can be identified as the selected feature. Similar to the above two meta-heuristics the final selected feature set is the one with the minimum cost (error of classification).

\section{Metaheuristic Feature Selection Pseudo Code and Their Parameters}

The above mentioned algorithms are used to select a given number of feature $\left(n_{s}\right)$ from all $\left(n_{T}\right.$ $=1886$ ) extracted features so that the error of classification is a minimum. In all the above mentioned algorithms the population size is taken as 5 . The detailed parameter values of the metaheuristic algorithms are given in table 2 which are fixed after several trial and errors. Sufficiently higher iteration (i.e. 1000) is taken due proper convergence of the metaheuristic algorithms. Same iteration and population value is taken for SA, PSO and ACO. In the first phase all integer values of $n_{s}=1$ to 20 is varied sequentially and the search continued for 1000 iteration in each case as the time constraint is less severe for small $n_{s}$ but chances of finding the best set is high. To find feature set of size more than 20 , the number of iteration is reduced to 100 keeping the population sizes same because the time requirement increases rapidly as $n_{s}$ increases. The algorithm used in the work is given below for PSO, ACO and SA.

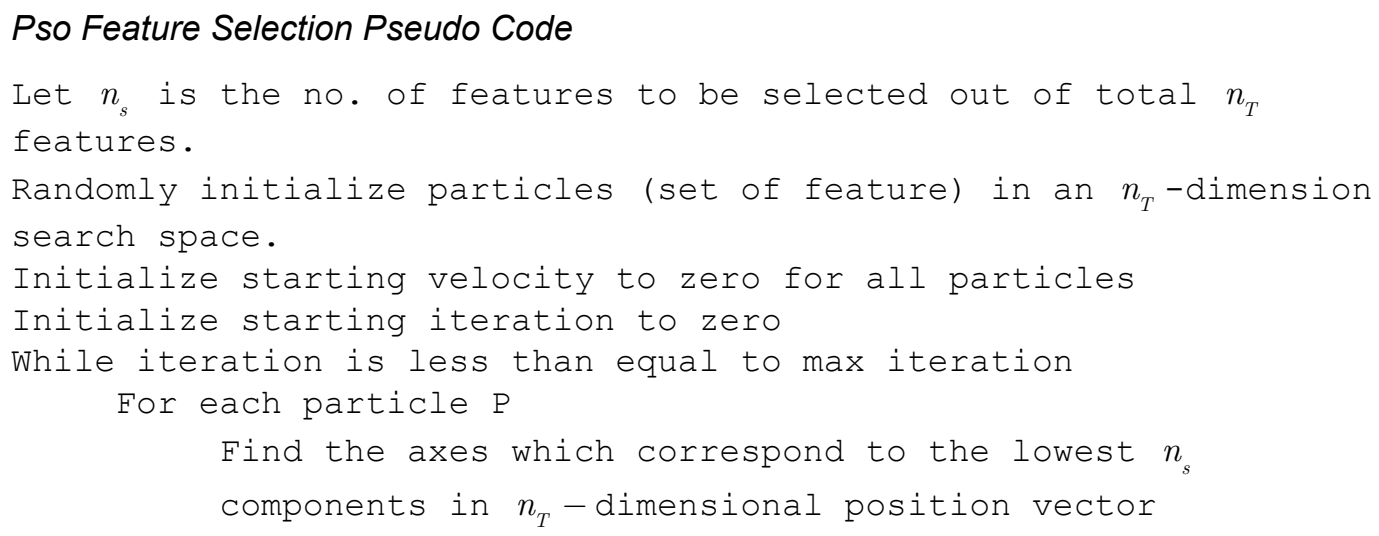


Choose the features corresponding to the above $n_{s}$ axes.

Execute MLP with chosen $n_{s}$ feature

Find out mean accuracy (fitness function) of 5-fold

cross-validation of the current particle position

Revise the PBes

EndFor

Revise the GBes

For each particle

Revise the particle velocity and position by PSO

position, velocity revise rule (1) and (2)

EndFor

Increment iteration

EndWhile

The features corresponding to the final Gbes position is the selection and the corresponding performance indicators are reported.

\section{Aco Feature Selection Pseudo Code}

Let $n_{s}$ is the no. of features to be selected out of total $n_{T}$ features.

Set initial pheromone and evaporation rate, pheromone exponential and heuristic weight

While iteration is less than equal to max iteration

For each particle (or ant)

Select a random node from a graph of $n_{T}$ nodes

Determine the sequence of nodes (tour). Probability of

selecting node $j$ after $i$ is proportional to the

pheromone intensity of edge $i-j$ using rule (3)

Choose the features corresponding to the first $n_{s}$ nodes

of the above sequence

Execute MLP with chosen $n_{s}$ feature

Find out mean accuracy (fitness function) of 5-fold

cross-validation of the current tour

If new Accuracy is better than the Accuracy found so far

Store the feature set as best feature set EndIf

EndFor

Revise pheromone intensity of each edge of the graph using rule (4) Increment iteration

EndWhile

The features corresponding best accuracy at the end of iterations is the selection and the corresponding performance indicators are reported.

\section{Sa Feature Selection Pseudo Code}

Let $n_{s}$ is the no. of features to be selected out of total $n_{T}$ features. Set an initial Temperature and reduction rate While iteration is less than equal to max iteration 


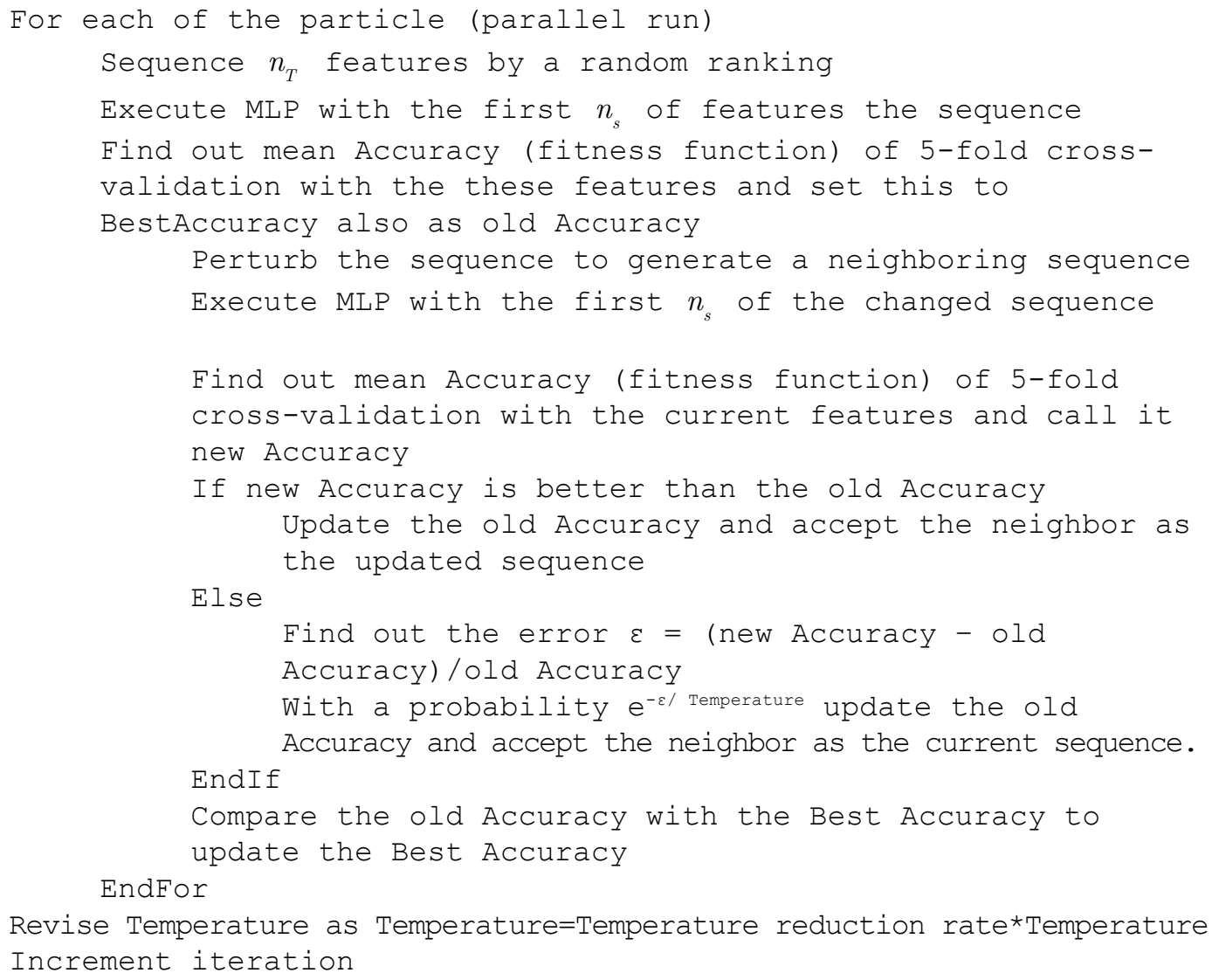

Revise Temperature as Temperature=Temperature reduction rate*Temperature Increment iteration

\section{EndWhile}

It is to be noted that although SA is not a collective search it is used in a batch mode. A number of parallel runs (particles) are executed at each temperature starting from random initial conditions. If the number of iteration is $m$ and number of particle is $n$ in the three feature selection algorithms PSO, ACO and SA then the worst case time complexity of all the three algorithm is $O\left(m^{*} n\right)$ i.e. $O\left(n^{2}\right)$ when $m$ is equal to $n$.

\section{CLASSIFIER USED}

In this section, a small discussion on two classifiers used is given along with the values of the relevant parameters.

\section{Support Vector Machine (SVM)}

SVM (Cortes \& Vapnik, 1995; Steve, 1998) is an extensively used supervised regression and classification algorithm. SVM, a non-probabilistic linear classifier, creates a single or multiple hyperplanes in an n-dimensional space for classification.

\section{Artificial Neural Network (ANN)}

ANN (Rumelhart, Hinton, \& Williams, 1986; Hagan, Demuth, \& Beale, 1996) is influenced by the working principle of biological neurons present in the human brain. It consists of one input and output layer and one/several hidden layers. Each layer has single or multiple numbers of neurons which can 
Table 2. Different metaheuristic algorithm parameters used in the experiment

\begin{tabular}{|l|l|}
\hline \multicolumn{1}{|c|}{ Algorithm } & \multicolumn{1}{c|}{ Value of the parameters } \\
\hline \multirow{3}{*}{ SA } & $\begin{array}{l}\text { Starting temperature: } 10 \\
\text { Temperature reduction rate: } 0.99 \\
\text { Iteration: } 1000 \text { for selecting features } 1 \text { to } 20, \text { after that iterations made to } 100 \text { to select the rest of the } \\
\text { features. } \\
\text { Population (No of parallel runs): } 5\end{array}$ \\
\hline \multirow{3}{*}{ ACO } & $\begin{array}{l}\text { Starting Ant Pheromone: } 1 \\
\text { Ant pheromone-exponential-weight: } 1 \\
\text { Rate of Ant Pheromone evaporation: } 0.05 \\
\text { Iteration: } 1000 \text { for selecting features } 1 \text { to } 20, \text { after that iterations made to } 100 \text { to select the rest of the } \\
\text { features. } \\
\text { Population (number of Ant): } 5\end{array}$ \\
\hline \multirow{3}{*}{ PSO } & $\begin{array}{l}\text { Starting inertia: } 0.729 \\
\text { Rate of inertia damping: } 1 \\
\text { Rate of individual particle learning: } 1.496 \\
\text { Rate of social learning: } 1.496 \\
\text { Value of random number ra1 and ra2: varies between } 0 \text { and } 1 \\
\text { Iteration: } 1000 \text { for selecting features } 1 \text { to } 20, \text { after that iterations made to } 100 \text { to select the rest of the } \\
\text { features. } \\
\text { Population: } 5\end{array}$ \\
\hline
\end{tabular}

receive a signal (real number) to perform a nonlinear function on its summated input and transmit the output to neurons in the next layer. Each time of the multiple iterations all the artificial neurons adjust their weights for the betterment of learning. Finally the output layer gives the probability of a particular sample to the output class level.

\section{Classification Algorithm Parameters}

In Figure 4 detailed steps of the present work are given in a flow diagram. After extracting 1886 features from 170 MED-NODE images the meta-heuristic algorithms (SA/ACO/PSO) are used to select an optimized set of features. The fitness function in SA, ACO and PSO is classification accuracy, which is calculated by MLP with fixed 10 hidden layer neurons and Levenberg-Marquardt training function and mean squared error cost function. After selecting the $n_{s}$ number of features of 170 samples of the binary class (100 malignant samples and benign 70 samples) are given to MLP and SVM classifiers separately and final classification accuracy is noted for both MLP and SVM. Same

Figure 4. Flow diagram of the proposed work

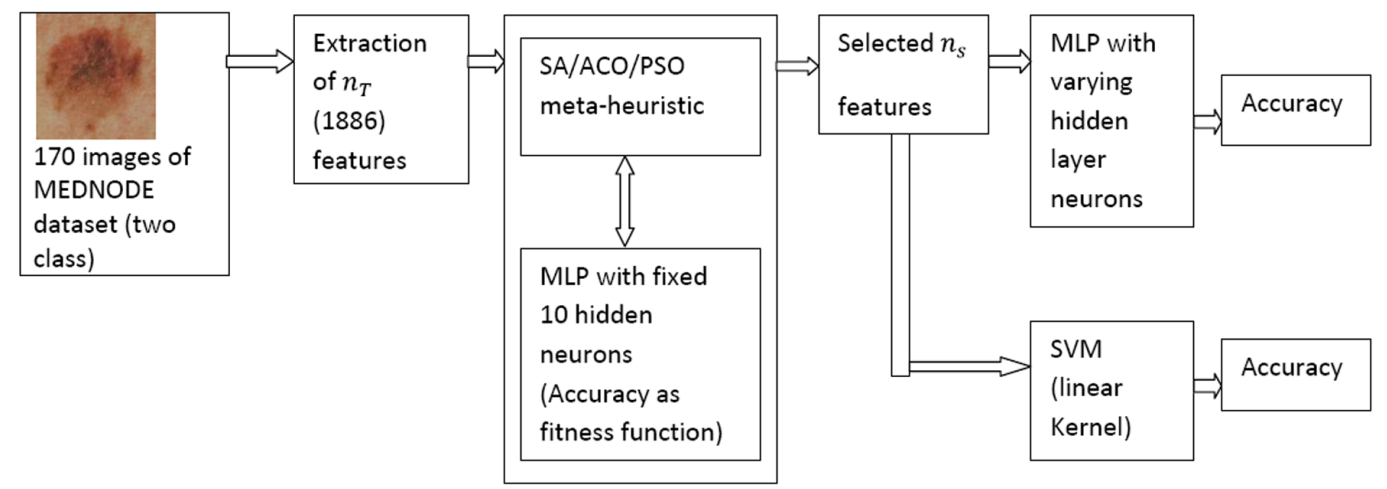


protocol is maintained in case of both the classifiers (SVM and ANN) in respect of training, validation and testing set. From the entire MED-NODE image set 70\% (119), 15\% (25) and 15\% (26) samples are exercised to train, validate and to test the system respectively. In this work 5-fold cross-validation is used where in each time different training, validation and testing sets are used and finally the average of test results is taken into consideration. The number of hidden layer neurons is changed from 1 to half the number of features used in the classification work as discussed by Stathakis (Stathakis, 2009). The best accuracy result thus obtained by varying hidden layer neurons is taken into consideration. In the SVM classifier linear kernel is used. The accuracy percentage is calculated as equation 5:

$$
\text { Accuracy }=\frac{N I C C}{T N T I}
$$

where:

NICC $=$ Number of Images Correctly Classified

and:

TNTI $=$ Total Number of Test Images

\section{RESULT ANALYSIS}

This proposed work is implemented in MATLAB software (Ver. R2018a) and an Intel Core i5-6500, 3.2 $\mathrm{GHz}$ processor with $8 \mathrm{~GB}-\mathrm{RAM}$ is used as hardware. Numbers of selected features, $\mathrm{n}_{\mathrm{s}}$ are varied sequentially using 3 metaheuristic algorithms PSO, ACO and SA. Initially all values between 1 to 20 are tried and then $n_{s}$ was increased with larger gaps. Total 35 different values of $n_{s}$ were explored to see their effects in the accuracy of classification the results. The results are shown in the following figures. The plot of number of features versus the classification accuracies are shown (figure 5 to figure 9) and to show variations of accuracies with small sets of features, accuracies versus feature set 1 to 20 are drawn separately in the same figure. In figure 5, figure 6 and figure 7 features are selected by PSO, ACO and SA respectively and the classifiers SVM and ANN. In figure 8 and figure 9 the relative comparison of classification accuracies for the three metaheuristic algorithms with ANN and SVM are shown respectively. It is found that almost all values of the $n_{s}$ PSO selected set performs better. It can be seen that only using 7 features selected by PSO an accuracy of $87.69 \%$ is found using ANN classifier, which is the highest accuracy (\%) using the smallest set of features with MED-NODE data set. With 16 features selected by ACO an accuracy of $86.15 \%$ is obtained using ANN. With 19 features selected by SA the accuracy is $82.3 \%$. With only 7 features selected by PSO, SVM gives an accuracy of $82.4 \%$. It can be found from the result that PSO performs better than ACO and SA. The results found from the ANN classifier are better than SVM classifier with mostly all sets of features. With all features the accuracy $85 \%$ is achieved in the present work.

In table 3 the best results found in the present work using the three metaheuristic algorithms are given with the classifier.

In table 4 the details about the 7 features are given, which are selected by PSO and gives a best classification accuracy of $87.69 \%$. It can be seen that out of these 7 features, 1 first order color feature is present and other 6 features belong to second order texture categories ( 2 of them are GLRLM and 4 of them are GLCM features). It is also to be noted that all the texture features are using the interpixel angle of $0^{\circ}$ degree. 
Figure 5. Accuracy (\%) versus $n_{s}$ PSO selected features for SVM and ANN classifier
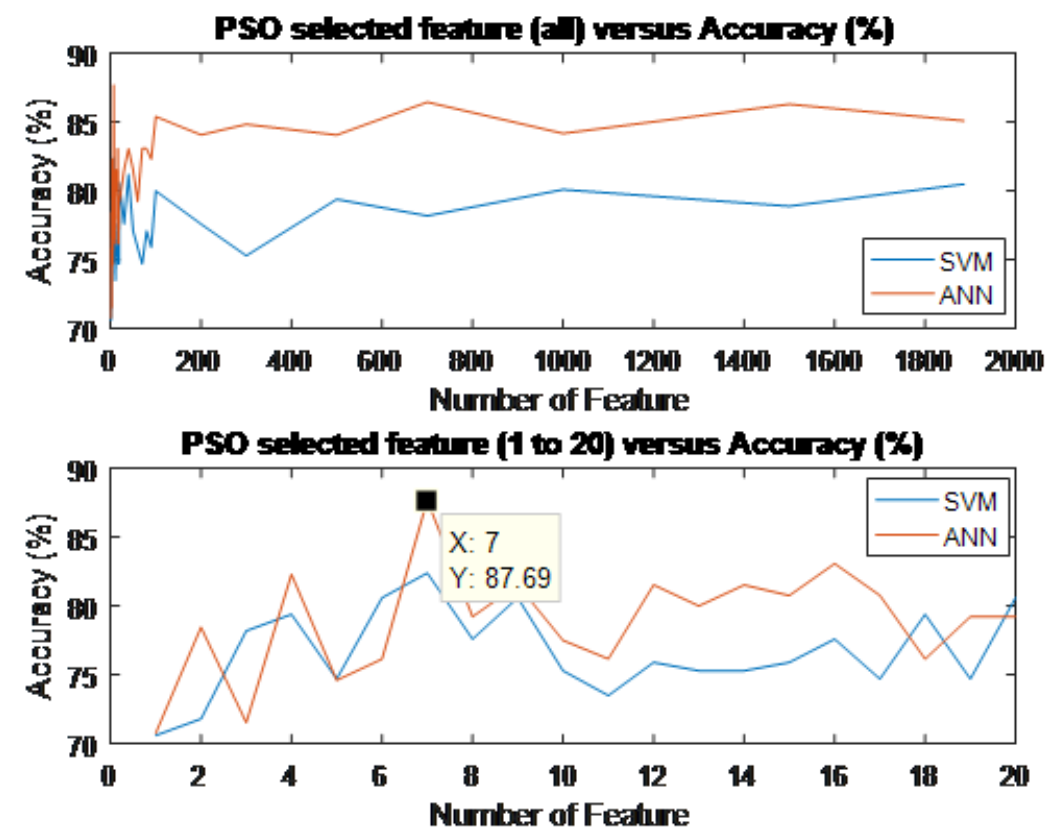

Figure 6. Accuracy (\%) versus ACO selected features for SVM and ANN classifier
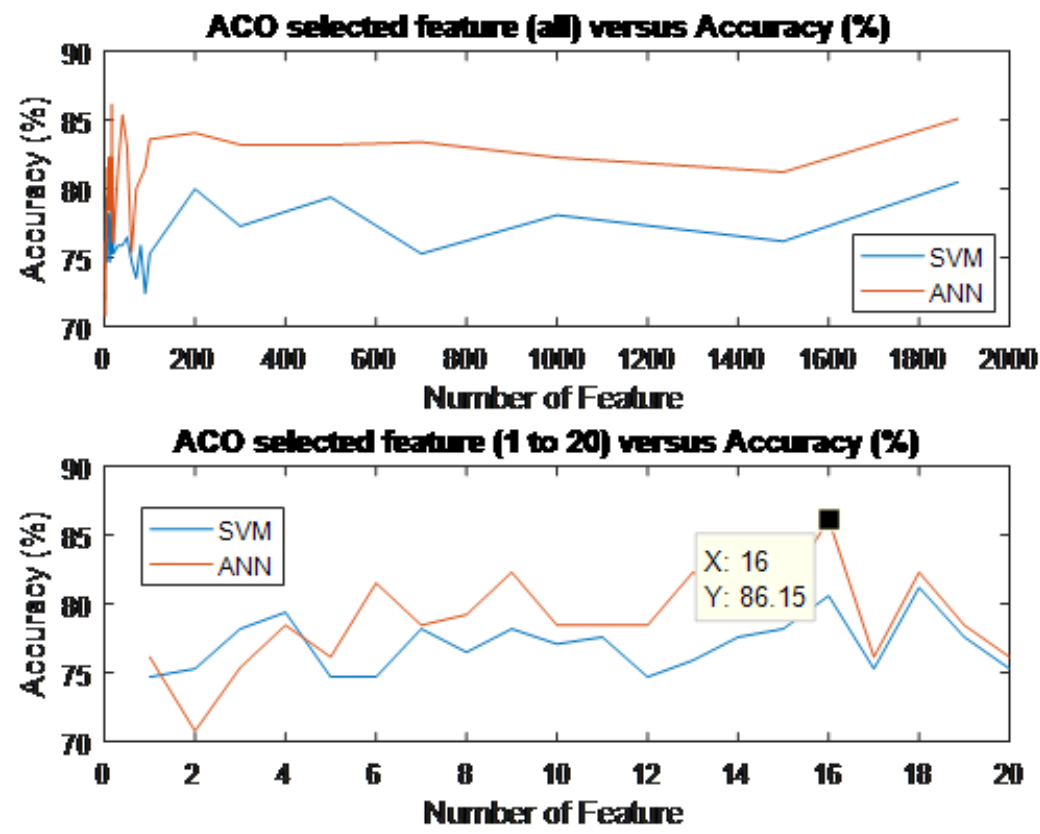
International Journal of Healthcare Information Systems and Informatics Volume $16 \cdot$ Issue 4

Figure 7. Accuracy (\%) versus SA selected features for SVM and ANN classifier
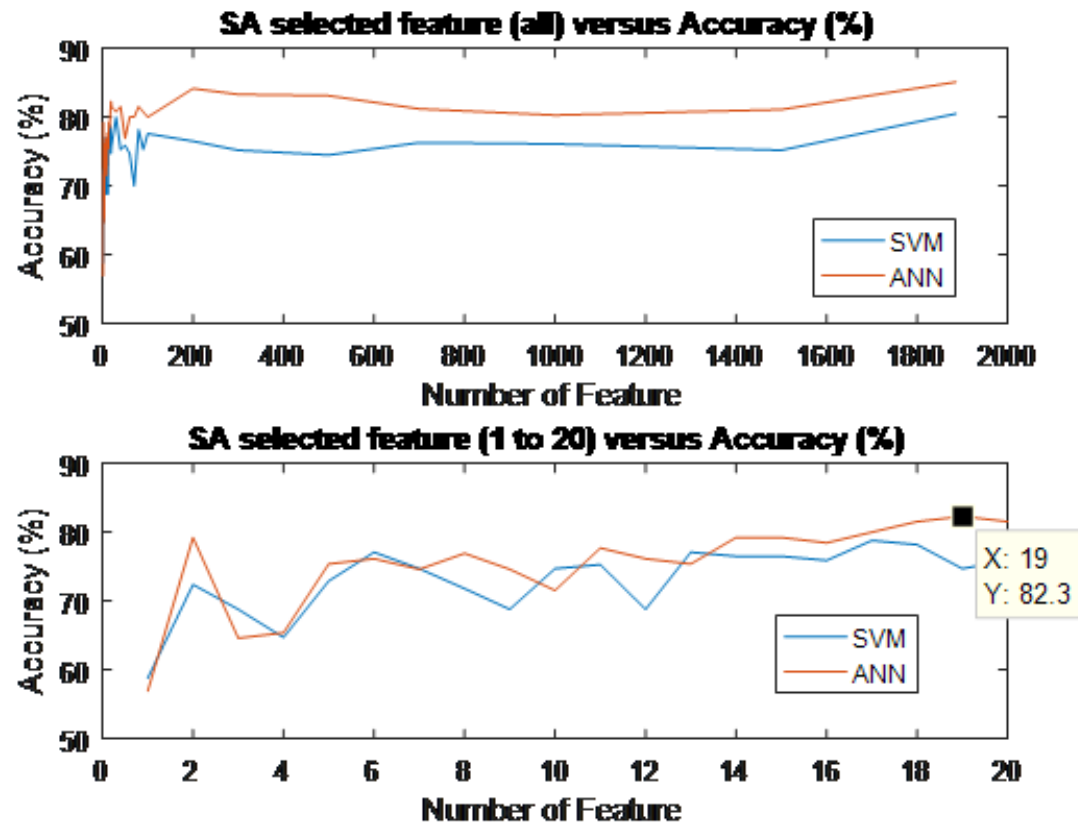

Figure 8. Accuracy (\%) versus features selected by PSO, ACO and SA with SVM classifier
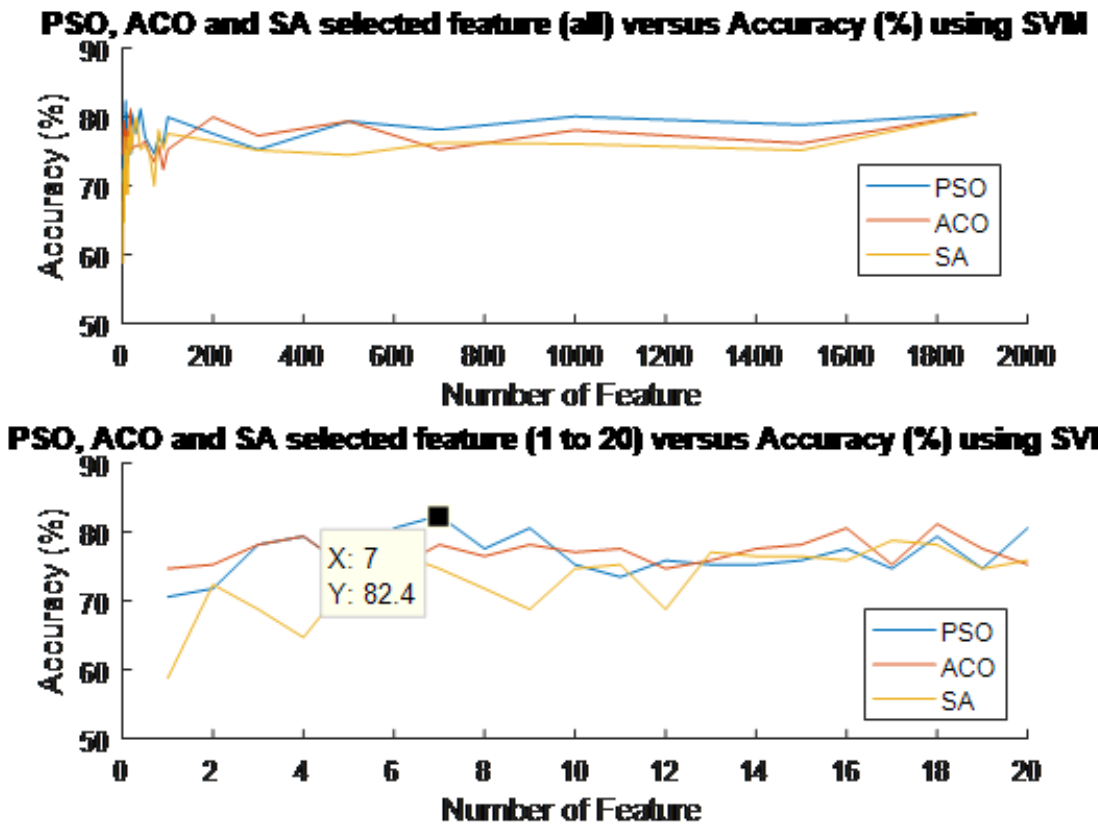
Figure 9. Accuracy (\%) versus features selected by PSO, ACO and SA with ANN classifier

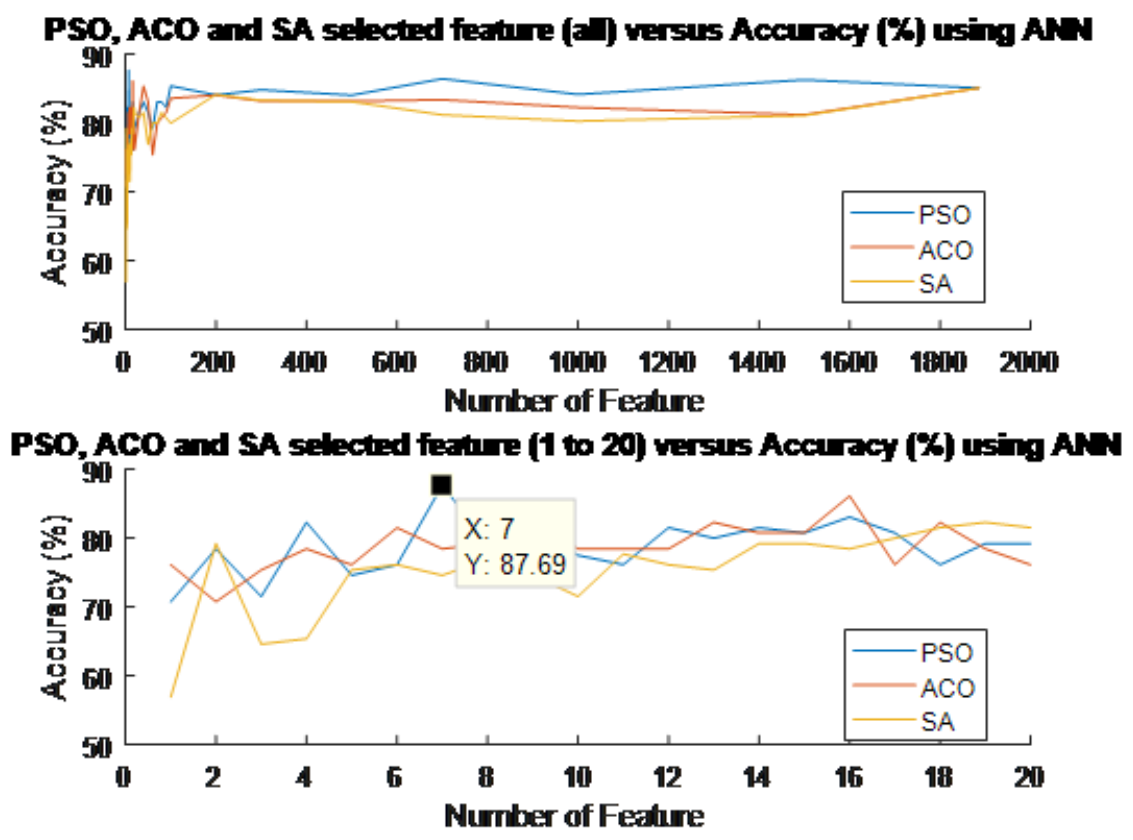

Table 3. Best results found in the present work using the three metaheuristic algorithms

\begin{tabular}{|c|c|c|c|}
\hline Metaheuristic Algorithm & Number of Features & Classifier Used & Accuracy Found \\
\hline PSO & 7 & ANN & $87.69 \%$ \\
\hline ACO & 16 & ANN & $86.15 \%$ \\
\hline SA & 19 & ANN & $82.3 \%$. \\
\hline
\end{tabular}

Table 4. Feature in details present in the best set of feature

\begin{tabular}{|c|c|c|c|c|c|}
\hline $\begin{array}{l}\text { Feature } \\
\text { Number }\end{array}$ & Feature Name & Color Channel & $\begin{array}{l}\text { Quantization } \\
\text { Level }\end{array}$ & $\begin{array}{c}\text { Inter-Pixel } \\
\text { Angel in } \\
\text { Degree }\end{array}$ & $\begin{array}{c}\text { Inter-Pixel } \\
\text { Distance }\end{array}$ \\
\hline 1770 & $\begin{array}{l}\text { Short-Run-Low-Gray-Level- } \\
\text { Emphasis (GLRLM) }\end{array}$ & Gray & 128 & 0 & 1 \\
\hline 1858 & Standard-Deviation & "V" of HSV & ---- & ---- & ---- \\
\hline 1247 & Entropy (GLCM) & "V" of HSV & 64 & 0 & 6 \\
\hline 1179 & Correlation (GLCM) & "H" of HSV & 64 & 0 & 3 \\
\hline 1694 & Sum-Variance (GLCM) & "Value" of HSV & 64 & 0 & 3 \\
\hline 769 & Sum-Average (GLCM) & "B" of RGB & 128 & 0 & 1 \\
\hline 1805 & $\begin{array}{l}\text { Gray-Level-Non-uniformity } \\
\text { (GLRLM) }\end{array}$ & Gray & 64 & 0 & 1 \\
\hline
\end{tabular}


Table 5. Result comparison of the proposed work with other related work using MED-NODE dataset

\begin{tabular}{|c|c|c|}
\hline Related Work & Feature Number \& Type & Accuracy (\%) Found \\
\hline (Spiros, et al., 2017) & 14 shape, color \& texture features & 76.2 \\
\hline (Mahecha, Parra \& Velandia, 2018) & Deep CNN features & 76.67 \\
\hline (Nazneen, Mandal, \& Puhan, 2018) & Deep CNN features & 77.1 \\
\hline $\begin{array}{l}\text { (Jafari, Samavi, Karimi, } \\
\text { Soroushmehr, Ward, \& Najarian, } \\
\text { 2016) }\end{array}$ & 10 hand-crafted features & 79 \\
\hline $\begin{array}{l}\text { (Giotis, Molders, Land, Biehl, } \\
\text { Jonkman, \& Petkov, 2015) }\end{array}$ & 675 hand-crafted features & 81 \\
\hline $\begin{array}{l}\text { (Mukherjee, Adhikari, \& Roy, } \\
\text { 2020). }\end{array}$ & 10 texture features & 83.33 \\
\hline $\begin{array}{l}\text { (Mukherjee, Adhikari, \& Roy, } \\
\text { 2018a) }\end{array}$ & $\begin{array}{l}1875 \text { shape, color } \& \text { texture features with } 4 \text { and } 8 \\
\text { quantization level }\end{array}$ & 85.9 \\
\hline $\begin{array}{l}\text { (Mukherjee, Adhikari, \& Roy, } \\
\text { 2020). }\end{array}$ & 25 PCA features & 87.18 \\
\hline $\begin{array}{l}\text { (Mukherjee, Adhikari, \& Roy, } \\
\text { 2018b) }\end{array}$ & $\begin{array}{l}1886 \text { shape, color \& texture features with } 64 \text { and } 128 \\
\text { quantization level }\end{array}$ & 91.02 \\
\hline Proposed work & 7 color \& texture features & 87.69 \\
\hline
\end{tabular}

\section{RESULT COMPARISON}

The table 5 represents the comparison between different related works with the MED-NODE dataset. The proposed work fares much better in respect of classification accuracy with lowest number of features than the other related works. All the work given in table 5 used computational facilities with the help of machine learning or deep learning. Brinker et al. (Brinker, Hekler, et al., 2019) showed that with the same MED-NODE dataset a group of 157 dermatologists achieved overall $89.4 \%$ and $64.4 \%$ sensitivity and specificity respectively with their domain knowledge. The proposed work achieved a similar level of accuracy. Mahecha et al. (Mahecha, Parra \& Velandia, 2018) and Nazneen et al. (Nazneen, Mandal, \& Puhan, 2018) used Deep CNN which is computational expensive. Spiros et al. (Spiros, et al., 2017) and Jafari et al. (Jafari, Samavi, Karimi, Soroushmehr, Ward, \& Najarian, 2016) used only 14 and 10 features respectively in their work which is very small in comparison with 675 features used by Giotis et al. (Giotis, Molders, Land, Biehl, Jonkman, \& Petkov, 2015). Mukherjee et al. (Mukherjee, Adhikari, \& Roy, 2020) used only 10 features. In the proposed work with only 7 features better accuracy is achieved in comparison with other work given in table 5 . Detailed discussions of all the papers are given in the literature survey section.

\section{CONCLUSION}

Ever increasing medical expenses in skin cancer makes life difficult for the patients and the family members. Early detection of melanoma skin cancer holds the major key behind the cure of the disease. In this work it is observed that the PSO feature selection algorithm is much better than the ACO and

SA feature selection algorithms. This result is expected where the $\frac{n_{s}}{n_{T}}$ the ratio is low. In case of ACO and SA when only a small number node of the route/sequence is selected for calculating the cost function, the costs of the subsequent routes/neighbors become almost independent. On the other 
hand extraction of less number features and classification using the same is computationally less time consuming. Keeping the above mentioned concept in mind the aim of the work is to find a small but effective feature subset for melanoma classification. PSO feature selection algorithm selects optimized feature sets (only 7 out of 1898 features) to provide best classification accuracy of $87.69 \%$. It can be seen that out of these 7 features, 1 first order color feature is present and other 6 features belong to second order texture categories ( 2 of them are GLRLM and 4 of them are GLCM features). This is noticeable that classification accuracy achieved in this work is considerably greater than the accuracies found previous with this dataset. The result found in one of the literature survey by Mukherjee et al. (Mukherjee, Adhikari, \& Roy, 2018b) has a higher accuracy rate of $91.02 \%$ but it requires 1886 number of features, which is about 270 times larger than the number of features used in the current work. So this work is computationally less expensive. It is also found that the texture features are the best to classify malignant and benign lesions as 6 of the 7 features are texture feature in the optimal feature set for the present work. Mukherjee et al. (Mukherjee, Adhikari, \& Roy, 2020) also found that most of the features are texture features that give better classification accuracy in their work with the same dataset. It is worth noting that parallel research work for melanoma detection in the medical and pathological field (Brinker \& Hekler, et al., 2019) is also ongoing. The authors have also done a comparative analysis of the result found in this work with the result found by the dermatologist. Recently Deep learning algorithms show promising performances in image processing research but due to huge computational time and essential usage of hardware like GPU the Deep learning application is not suitable for Smartphone or PDA application. The results of this work show better performances in comparison to the related Deep learning implementations.

\section{ACKNOWLEDGMENT}

The authors are grateful to Mr. Subhamon Supantha of University of Hyderabad, India for going through the manuscript of this paper and giving some useful suggestions to improve the presentation quality of the article. 


\section{REFERENCES}

Apalla, Z., Lallas, A., Sotiriou, E., Lazaridou, E., \& Ioannides, D. (2017). Epidemiological trends in skin cancer. Dermatology Practical \& Conceptual, 7(2), 1-6. doi:10.5826/dpc.0702a01 PMID:28515985

Arora, S., Singh, H., Sharma, M., Sharma, S., \& Anand, P. (2019). A New Hybrid Algorithm based on Grey Wolf Optimization and Crow Search Algorithm for unconstrained function optimization and feature selection. IEEE. doi:10.1109/ACCESS.2019.2897325

Aurora, S., Begoña, A., \& Carmen, S. (2014). Pattern Analysis in Dermoscopic Images. In J. Scharcanski (Ed.), Computer Vision Techniques for the Diagnosis of Skin Cancer. Series in BioEngineering. doi:10.1007/978-3642-39608-3_2

Blum, C. (2005). Ant colony optimization: Introduction and recent trends. Physics of Life Reviews, 2(4), 353-373. doi:10.1016/j.plrev.2005.10.001

Brinker, T., Hekler, A., Hauschild, A., Berking, C., Schilling, B., Enk, A. H., Haferkamp, S., Karoglan, A., von Kalle, C., Weichenthal, M., Sattler, E., Schadendorf, D., Gaiser, M. R., Klode, J., \& Utikal, J. S. (2019). Comparing artificial intelligence algorithms to 157 German dermatologists. the melanoma classification benchmark. European Journal of Cancer, 111, 30-37. doi:10.1016/j.ejca.2018.12.016 PMID:30802784

Clausi, D. A. (2002). An analysis of co-occurrence texture statistics as a function of grey level quantization. Canadian Journal of Remote Sensing, 28(1), 45-62. doi:10.5589/m02-004

Codella, N. C. F., Nguyen, Q.-B., Pankanti, S., Gutman, D. A., Helba, B., Halpern, A. C., \& Smith, J. R. (2017). Deep learning ensembles for melanoma recognition in dermoscopy images. IBM Journal of Research and Development, 61(4), 5:1-5:15. Advance online publication. doi:10.1147/JRD.2017.2708299

Cortes, C., \& Vapnik, V.N. (1995). Support-vector networks. Machine Learning, 20(3), 273-297. .10.1007/ BF00994018

Dorigo, M., Maniezzo, V., \& Colorni, A. (1996). Ant System: Optimization by a colony of cooperating agents. IEEE Trans Syst Man Cybernet Part B, 26(1), 29-41. doi:10.1109/3477.484436 PMID:18263004

Esteva, A., Kuprel, B., Novoa, R. A., Ko, J., Swetter, S. M., Blau, H. M., \& Thrun, S. (2017). Dermatologist-level classification of skin cancer with deep neural networks. Springer Nature. doi:10.1038/nature21056

Fleischer, M. (1995). Simulated annealing: past, present, and future. Winter Simulation Conference Proceedings, 155-161.

Fong, S., Wong, R., \& Vasilakos, A. V. (2016). Accelerated PSO Swarm Search Feature Selection for Data Stream Mining Big Data. IEEE Transactions on Services Computing, 9(1), 33-45. doi:10.1109/TSC.2015.2439695

Giotis, I., Molders, N., Land, S., Biehl, M., Jonkman, M. F., \& Petkov, N. (2015). MED-NODE: A computerassisted melanoma diagnosis system using non-dermoscopic images. Expert Systems with Applications, 42(19), 6578-6585. doi:10.1016/j.eswa.2015.04.034

Gorai, S., \& Chatterjee, S. (2019). Extraction of features from cross correlation in space and frequency domains for classification of skin lesions. Biomedical Signal Processing and Control, 53.

Hagan, M. T., Demuth, H. B., \& Beale, M. H. (1996). Neural Network Design. PWS Publishing.

Haralick, R. M., Shanmugam, K., \& Dinstein, I. (1973). Textural Features of Image Classification. IEEE Transactions on Systems, Man, and Cybernetics, SMC-3(6), 610-621. doi:10.1109/TSMC.1973.4309314

Ighazran, H., Alaoui, L., \& Boujiha, T. (2018) Metaheuristic and Evolutionary Methods for Feature Selection in Sentiment Analysis (a Comparative Study). International Symposium on Advanced Electrical and Communication Technologies (ISAECT), 1-6. doi:10.1109/ISAECT.2018.8618799

Jafari, M. H., Samavi, S., Karimi, N., Soroushmehr, S. M. R., Ward, K., \& Najarian, K. (2016). Automatic detection of melanoma using broad extraction of features from digital images. 38th Annual International Conference of the IEEE Engineering in Medicine and Biology Society (EMBC). doi:10.1109/EMBC.2016.7590959

Kennedy, J., \& Eberhart, R. C. (1995). Particle Swarm Optimization. Proceedings of the IEEE international conference on neural networks IV, 1942-1948. 
Laskaris, N., Ballerini, L., Fisher, R. B., Aldridge, B., \& Rees, J. (2010). Fuzzy Description of Skin Lesions. SPIE Proceedings, 7627.

Leiter, U., Eigentler, T., \& Garbe, C. (2014). Epidemiology of skin cancer. Advances in Experimental Medicine and Biology, 810, 120-140.

Li, Y. (2016). Skin Cancer Detection and Tracking using Data Synthesis and Deep Learning. NIPS 2016 Workshop on Machine Learning for Health, Barcelona, Spain.

Mahecha, M. S. S., Parra, O. J. S., \& Velandia, J. B. (2018). Design of a System for Melanoma Detection Through the Processing of Clinical Images Using Artificial Neural Networks. In Challenges and Opportunities in the Digital Era. I3E 2018. Lecture Notes in Computer Science, (vol. 11195). Springer.

Matthews, N. H. (2017). Epidemiology of Melanoma. In Cutaneous Melanoma: Etiology and Therapy. Codon Publications.

McDonagh, S., Fisher, R.B., \& Rees, J. (2008). Using 3D information for classification of non-melanoma skin lesions. Medical Image Understanding and Analysis, 164-168.

Morton, C. A., \& Mackie, R. M. (1998). Clinical accuracy of the diagnosis of cutaneous malignant melanoma. British Journal of Dermatology, 138(2), 283-287. doi:10.1046/j.13652133.1998.02075.x

Mukherjee, S., Adhikari, A., \& Roy, M. (2018a). Malignant Melanoma Detection using Multi Layer Perceptron with Optimized Network Parameter Selection by PSO. In 1st International Conference on Contemporary Advances in Innovative \& Applicable Information Technology. Springer. doi:10.1007/978-981-13-1540-4_11

Mukherjee, S., Adhikari, A., \& Roy, M. (2018b). Melanoma Identification using MLP with Parameter Selected by Metaheuristic Algorithms. In Intelligent Innovations in Multimedia Data Engineering and Management. IGI Global. doi:10.4018/978-1-5225-7107-0.ch010

Mukherjee, S., Adhikari, A., \& Roy, M. (2018c). Malignant Melanoma Classification using Cross Platform Dataset with Deep Learning CNN Architecture. In Second International Symposium on Signal and Image Processing (ISSIP), Recent Trends in Signal and Image Processing. Advances in Intelligent Systems and Computing, (vol. 922). Springer. doi:10.1007/978-981-13-6783-0_4

Mukherjee, S., Adhikari, A., \& Roy, M. (2018d). Malignant Melanoma Identification using Best Visually Imperceptible Features from Dermofit Dataset. 1st International Conference on Emerging Trends in Engineering and Science, Proceedings of ETES 2018, Lecture Notes in Network and System. doi:10.1007/978-981-13-3122-0_25

Mukherjee, S., Adhikari, A., \& Roy, M. (2020). Malignant Melanoma Detection using Multi Layer Preceptron with Visually Imperceptible Features and PCA Components from MED-NODE Dataset. International Journal of Medical Engineering and Informatics, 12(2), 151-168. doi:10.1504/IJMEI.2020.106899

Mukherjee, S., Adhikari, A., \& Roy, M. (in press). Melanoma Detection by Meta-heuristically Optimized MLP Parameters using Non-Dermatoscopy Images. International Journal of Applied Metaheuristic Computing .

Naeini, A. A., Babadi, M., Mirzadeh, S. M. J., \& Amini, S. (2018). Particle Swarm Optimization for Object-Based Feature Selection of VHSR Satellite Images. IEEE Geoscience and Remote Sensing Letters, 15(3), 379-383. doi:10.1109/LGRS.2017.2789194

Nazneen, N.S., Mandal, B., \& Puhan, N.B. (2018). Deep residual network with regularised fisher framework for detection of melanoma. IET Computer Vision.

Otsu, N. (1979). A threshold selection method from gray-level histograms. IEEE Transactions on Systems, Man, and Cybernetics, 9(1), 62-66. doi:10.1109/TSMC.1979.4310076

Pereira, S. M., Marco, A., Frade, C., Rangaraj, M., \& Azevedo-Marques, P. M. (2013). Classification of Color Images of Dermatological Ulcers. IEEE Journal of Biomedical and Health Informatics, 17(1).

Rumelhart, D. E., Hinton, G. E., \& Williams, R. J. (1986). Learning representations by back-propagating errors. Nature, 323(6088), 533-536. doi:10.1038/323533a0

Rutenbar, R. A. (1989). Simulated annealing algorithms: An overview. IEEE Circuits and Devices Magazine, 5(1), 19-26.

Seijas, L.M. (2015). Metaheuristics for feature selection in handwritten digit recognition. 2015 Latin America Congress on Computational Intelligence (LA-CCI), 1-6. doi: 10.1109/LA-CCI.2015.7435975 
Soh, L., \& Tsatsoulis, C. (1999). Texture Analysis of SAR Sea Ice Imagery Using Gray Level Co-Occurrence Matrices. IEEE Transactions on Geoscience and Remote Sensing, 37(2).

Spiros, K. (2017). Adaptable Pattern Recognition System for discriminating Melanocytic Nevi from Malignant Melanomas using plain photography images from different image databases. International Journal of Medical Informatics, 105. Advance online publication. doi:10.1016/j.jjmedinf.2017.05.016

Stathakis, D. (2009). How many hidden layers and nodes? International Journal of Remote Sensing, 30(8), 2133-2147. doi:10.1080/01431160802549278

Steve, R. G. (1998). Support Vector Machines for Classification and Regression. Technical Report. University of Southampton.

Tan, T. Y., Zhang, L., \& Jiang, M. (2016) An Intelligent Decision Support System for Skin Cancer Detection from Dermoscopic Images. Proceedings of the 12th International Conference on Natural Computation, Fuzzy Systems and Knowledge Discovery (ICNC-FSKD), 2194-2199. doi:10.1109/FSKD.2016.7603521

Tang, X. (1998). Texture Information in Run-Length Matrices. IEEE Transactions on Image Processing, 7(11), 1602-1609.

Thanh-Toan, D. (2018). Accessible Melanoma Detection using Smartphones and Mobile Image Analysis. IEEE Transactions on Multimedia, 20(10), 2849-2864. doi:10.1109/TMM.2018.2814346

Valle, E., Fornaciali, M., Menegola, A., \& Tavares, J. (2017). Data, Depth, and Design: Learning Reliable Models for Melanoma Screening. IEEE Journal of Biomedical and Health Informatics.

Wang, S., Zhang, Y., \& Ji, G. (2015). A Comprehensive Survey on Particle Swarm Optimization Algorithm and Its Applications. Mathematical Problems in Engineering. 10.1155/2015/931256

Xiao, F., \& Wu, Q. (2020). Visual saliency based global-local feature representation for skin cancer classification. IET Image Processing, 14. Advance online publication. doi:10.1049/iet-ipr.2019.1018

Yeh, J., \& Chan, S. (2017). Population-based metaheuristic approaches for feature selection on mammograms. 2017 IEEE International Conference on Agents (ICA), 140-144. doi:10.1109/AGENTS.2017.8015321

Soumen Mukherjee did his B.Sc (Physics Honours) from Calcutta University, M.C.A. from Kalyani University and ME in Information Technology from West Bengal University of Technology. He is the silver medalist in ME examination from the university. He has done his Post-Graduate Diploma in Business Management from Institute of Management Technology, Center of Distance Learning, Ghaziabad. He is now working as an Assistant Professor in RCC Institute of Information Technology, Kolkata. He has more than 17 years teaching experience in the field of Computer Science and Application. He has over 40 research paper published in different National and International Journal and Conferences and Book Chapter. He has total 9 chapters in different books under International Publisher. He has contributed in over 20 internationally acclaimed books in the field of Computer Science and Engineering. He has got two best paper awards in the conference and seminar. He has also edited 3 books. His research fields are Image Processing, Machine Learning and Pedagogy. He is a life member of several institutions like IETE, CSI, ISTE, FOSET, etc.

Arunabha Adhikari completed his M.Sc in Physics and Ph.D. from Calcutta University. He worked in SINP, Kolkata as a senior research fellow. His preliminary research was in Biophysics. His Ph.D. research topic was theoretical simulation of Calcium Action Potential. He worked in the laboratories of electrophysiology in IISc, Bangalore, India and Dept. of Physiology, University of Saarland, Germany. He has 21 years of teaching experience and presently working as an Associate Professor in the West Bengal State University. His present research domains are computational physics and neuroscience and application of ANN in medical image processing.

Madhusudan Roy acquired both his masters in Physics and Ph.D. degrees from the University of North Bengal, India in the year 1982 and 1989 respectively. He joined Indian Association for the Cultivation of Science, Kolkata, India as a Research Associate. During his tenure at Cultivation of Science, he worked on photoreaction dynamics of some organic compounds using Laser Raman Spectroscopy. Then he took up a job in the NEHU, India as a faculty in 1989, and in 2002, he joined SINP, Kolkata. He took part in many interdisciplinary research activities that includes medical image analysis. Now he retired from SINP. 